Boletín de la Sociedad Geológica Mexicana

VOLUMEN 64, NÚM. 1, 2012, P. 61-70

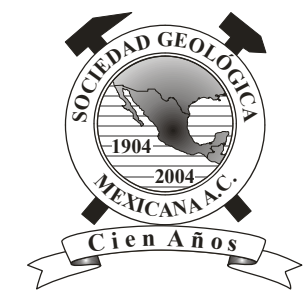

\title{
Calcretas pedogénicas en el Cretácico Tardío de Michoacán, México
}

\author{
Rosa E. Tovar ${ }^{1, *}$, David E. Fastovsky², M. Benammi ${ }^{3}$ \\ ${ }^{1}$ Posgrado en Ciencias de la Tierra, Universidad Nacional Autónoma de México, Ciudad Universitaria, México, D.F., 04510 \\ ${ }^{2}$ Department of Geosciences, University of Rhode Island, Kingston, RI, 02881 \\ ${ }^{3}$ Université de Poitiers, 40 Avenue du Recteur Pineau, Poitiers, France, 86022 \\ *rosae_tovar@yahoo.com.mx
}

\begin{abstract}
Resumen
El estudio de los paleosuelos, junto con la sedimentología, son herramientas útiles en la reconstrucción ambiental de los paisajes antiguos; asimismo han sido utilizados como marcadores estratigráficos para hacer correlaciones locales o para conocer tasas de sedimentación. Los paleosuelos más antiguos corresponden al Precámbrico, sin embargo han sido más estudiados los del Cuaternario. El objetivo del presente trabajo es dar una revisión acerca del uso y aplicaciones de los paleosuelos, con un mayor énfasis en el estudio de las calcretas pedogenéticas y presentar un estudio de caso perteneciente a la barranca Los Bonetes, Michoacán. La barranca Los Bonetes, localizada en el sur-centro de México, contiene sedimentos volcaniclásticos de edad Cretácico Tardío y los hadrosaurios más antiguos conocidos en México. Dentro de esta sucesión volcaniclástica se localizan paquetes de calcretas que, debido a sus características macro- y microestructurales, como son la presencia de raíces y las texturas alfa y beta, fueron reconocidas como de origen pedogenético. Con base en los requerimientos ambientales para la formación de este tipo de paleosuelos, interpretamos que el clima en la región debió ser árido o semiárido durante los periodos de no depósito durante el Cretácico. La importancia de este estudio radica en que es uno de los pocos trabajos en México en el cual se estudian paleosuelos precuaternarios para hacer inferencias climáticas.
\end{abstract}

Palabras clave: paleosuelos cretácicos, calcretas, paleoambiente, México, barranca Los Bonetes, dinosaurios.

\begin{abstract}
Paleosols and sedimentological studies are useful tools in the reconstruction of past environments; they are also used as stratigraphic markers for correlation in small areas or for determining the rate of deposition. The oldest known paleosols are from the Precambrian, however the most studied are those from the Quaternary. The purpose of this work is to give a brief review of the uses and applications of paleosols, with a special emphasis on pedogenic calcretes, concluding with a case study in the Barranca Los Bonetes, Michoacán. Barranca Los Bonetes, in the south-center of Mexico, bears a volcaniclastic succession of Late Cretaceous age and the oldest hadrosaurs known in Mexico. The volcaniclastic succession contains pedogenic calcretes, identified as such by macro- and microstructural characteristics such as roots, and alpha and beta textures. Based on the environmental requirements for the formation and accumulation of calcareous paleosols, we infer an arid or semi-arid climate in the study area during the periods of non-deposition during the Cretaceous. This study is, to date, one of the few studies in Mexico in which pre-Quaternary paleosols have been used as a tool for reconstructing past environments.
\end{abstract}

Keywords: Cretaceous paleosols, calcretes, paleoenvironment, Mexico, barranca Los Bonetes, dinosaurs. 


\section{Introducción}

Los paleosuelos, que en definición son restos de antiguos suelos (Retallack, 2001), se formaron por la interacción teórica de 5 factores: biota, topografía, clima, material parental y tiempo (Jenny, 1941). Tienden a formarse en periodos de estabilidad del paisaje e incluso se pueden formar en zonas terrestres donde sigue habiendo agradación, mientras ésta no impida la pedogénesis (Kraus, 1999).

Los paleosuelos han sido reconocidos desde el Precámbrico (Buick et al., 1995; Sreenivas et al., 2001) hasta el presente y han sido usados como marcadores estratigráficos, ya que pueden correlacionarse en áreas no muy extensas. Asimismo han sido usados para conocer la tasa de sedimentación presente en un área con base en el grado de desarrollo del paleosuelo; en el reconocimiento de incisiones en los valles y terrazas que son resultado de los cambios eustáticos; y comúnmente se utilizan para reconstruir los climas pasados, pudiéndose incluso estimar la tasa media de precipitación anual y la temperatura media anual (Kraus, 1999; Paik et al., 2001; Lee et al., 2003). Los paleosuelos también han sido utilizados para hacer inferencias o reconocer rasgos antiguos acerca de la colonización de los primeros organismos en tierra firme y han ayudado a reconocer algunos nidos de insectos como termitas, abejas y escarabajos (Retallack, 1985).

O'Brien y Stout (1978) han mostrado que los isótopos de carbono estables $\left({ }^{12} \mathrm{C} \mathrm{y}{ }^{13} \mathrm{C}\right)$ se concentran en las capas superficiales del suelo y que a mayor profundidad del suelo, el porcentaje de carbono tiende a disminuir. Krull y Retallack (2000) utilizando dicho modelo determinaron las condiciones y los tiempos de formación de los suelos presentes durante el Pérmico, el Permo-Triásico y el Triásico Temprano en la Antártida.

Los paleosuelos se reconocen en campo a través de tres características: trazas de raíces, horizontes y estructura de suelo (Retallack, 2001). Sin embargo, en suelos tan antiguos como los del Precámbrico (Buick et al., 1995; Sreenivas et al., 2001) y Paleozoico temprano (Retallack et al., 2003), la presencia de raíces no es un factor determinante, debido a que todavía no evolucionaban las plantas vasculares (Stanley, 2008).

Los paleosuelos no pueden ser estudiados de la misma forma que los suelos actuales debido a la alteración que han sufrido por el enterramiento y pérdida de su biota (Retallack, 1985). La determinación de los suelos actuales se basa en parámetros tales como la capacidad de intercambio catiónico, densidad aparente, régimen de humedad, constituyentes orgánicos en el perfil (p.ej. proporción de musgos), etc. Este tipo de características físicas y químicas no son conservadas en el registro geológico, por lo que Mack et al. (1993) han creado una clave basada en seis características o procesos: contenido de materia orgánica, horizontes presentes, condiciones redox, alteración mineral in situ, iluviación de compuestos o minerales insolubles y acumulación de minerales solubles. Sin embargo, su uso no ha sido muy favorecido debido a que algunos autores, como Retallack et al. (1993), han argumentado que no es clara la base de la clasificación, si es puramente descriptiva o está basada en la genética. Asimismo argumentan que el uso de las clasificaciones actuales en la determinación de los paleosuelos favorece la interpretación paleoambiental bajo el supuesto de que los procesos que actúan en los suelos actuales son los mismos que actuaron en el pasado. Por último, mencionan que si se acepta una clasificación exclusiva entre paleoedafólogos se perdería el lenguaje que se tiene en común con los edafólogos.

\subsection{Calcretas pedogenéticas}

El término calcreta o caliche ha sido usado para referirse a concreciones calcáreas que se desarrollan en suelos o en alteritas (sensu Meyer, 1997), aunque Wright y Tucker (1991) incluyen también a los estratos y sedimentos donde el nivel freático vadoso y somero se encuentra saturado en carbonato de calcio. Las calcretas se forman por la precipitación y cementación de cristales de calcita que empiezan a invadir poros y fisuras desplazando los elementos existentes (Meyer, 1997). Varían desde nodulares y calcáreas hasta altamente endurecidas debido al desplazamiento y/o reemplazamiento por la introducción de carbonatos vadosos en grandes o bajas cantidades dentro de suelos, rocas o sedimentos contenidos dentro de un perfil (Watts, 1980).

Se pueden formar en zonas de circulación de agua freática, pero generalmente es más común que se formen en ambientes pedológicos. En raras ocasiones, dentro de una misma región, ambos tipos de calcretas pueden formarse (Alonso-Zarza, 2003). La forma en que se reconocen ambos tipos de calcretas, las pedogenéticas y las no pedogenéticas, es a través de las relaciones de campo, tales como la posición inmediata por encima de una capa relativamente impermeable o estrato, así como la presencia o ausencia de fábricas alfa y beta observadas en lámina delgada (Rasbury et al., 2006).

Las calcretas que se encuentran en aguas subterráneas son de origen no pedogenético; se forman en la zona freática y son típicamente calizas lineales o tabulares que contienen principalmente fábricas alfa. Las texturas alfa incluyen los cristales de calcita rómbica, fábrica micrítica densa, granos de sedimento flotantes, grietas, cristalaria compleja, que es característica de crecimiento desplazante, y exhibe la ausencia de características biogénicas (Rasbury et al., 2006).

Las calcretas pedogenéticas se forman debido a una amplia variedad de procesos, los cuales comúnmente resultan de la precipitación de sales debido a evaporación en lugares cercanos a la superficie. El carbonato precipitado más común dentro de las unidades pedológicas, en general, es una calcita micrítica baja en magnesio. Los cristales individuales varían en tamaño desde la micrita hasta cristales de calcita con crecimiento de espadas de más de $2.5 \mathrm{~cm}$ de longitud. La morfología de los cristales varía entre agujas, 
espadas, calcita ecuante y en bloques (Chafetz et al., 1985).

Estas calcretas están atribuidas a condiciones de aridez y contienen fábricas alfa y beta y la extensión de cada tipo depende principalmente de las condiciones climáticas e hidrológicas. Las texturas beta contienen características que indican influencia biogénica tales como fábrica septal alveolar, túbulos calcificados, coberturas microbiales y Microcodium, una microestructura enigmática de calcita (Košir, 2004; Rasbury et al., 2006).

Uno de los tantos usos que se le han dado a las calcretas pedogenéticas es el de determinar la concentración de $\mathrm{CO}_{2}$ en la atmósfera e inferir los cambios en la misma durante diferentes eras geológicas (Ghosh et al., 2001; Nordt et al., 2002).

\section{Caso de estudio: barranca Los Bonetes}

Los depósitos sedimentarios continentales de edad cretácica son poco conocidos en el sur-centro de México y más raros han sido los hallazgos de restos de dinosaurios dentro de ellos, por lo que su descubrimiento en el depósito continental de la barranca Los Bonetes es importante para conocer los ambientes de depósito (Benammi et al., 2005; Tovar et al., 2009).

El objetivo de este trabajo es dar a conocer las calcretas pedogenéticas presentes en la sucesión volcaniclástica de la barranca Los Bonetes y la información paleoambiental que aportan en la determinación climática de la porción sur-centro de México durante el Cretácico Tardío.

\subsection{Descripción del área de estudio}

\subsubsection{Ubicación}

La barranca Los Bonetes se localiza sobre la carretera federal No. 51 dentro del estado de Michoacán, a 1903' latitud norte y $100^{\circ} 42^{\prime}$ longitud oeste, entre los municipios de Tiquicheo de Nicolás Romero y Tuzantla (Figura 1), perteneciendo a este último.

\subsection{Marco geológico regional}

La sucesión sedimentaria presente en la barranca Los Bonetes sobreyace una sección del Terreno Guerrero, cuya edad abarca desde el Jurásico Tardío hasta el Cretácico Tardío (Cenomaniano; Ortiz-Hernández et al., 2002;
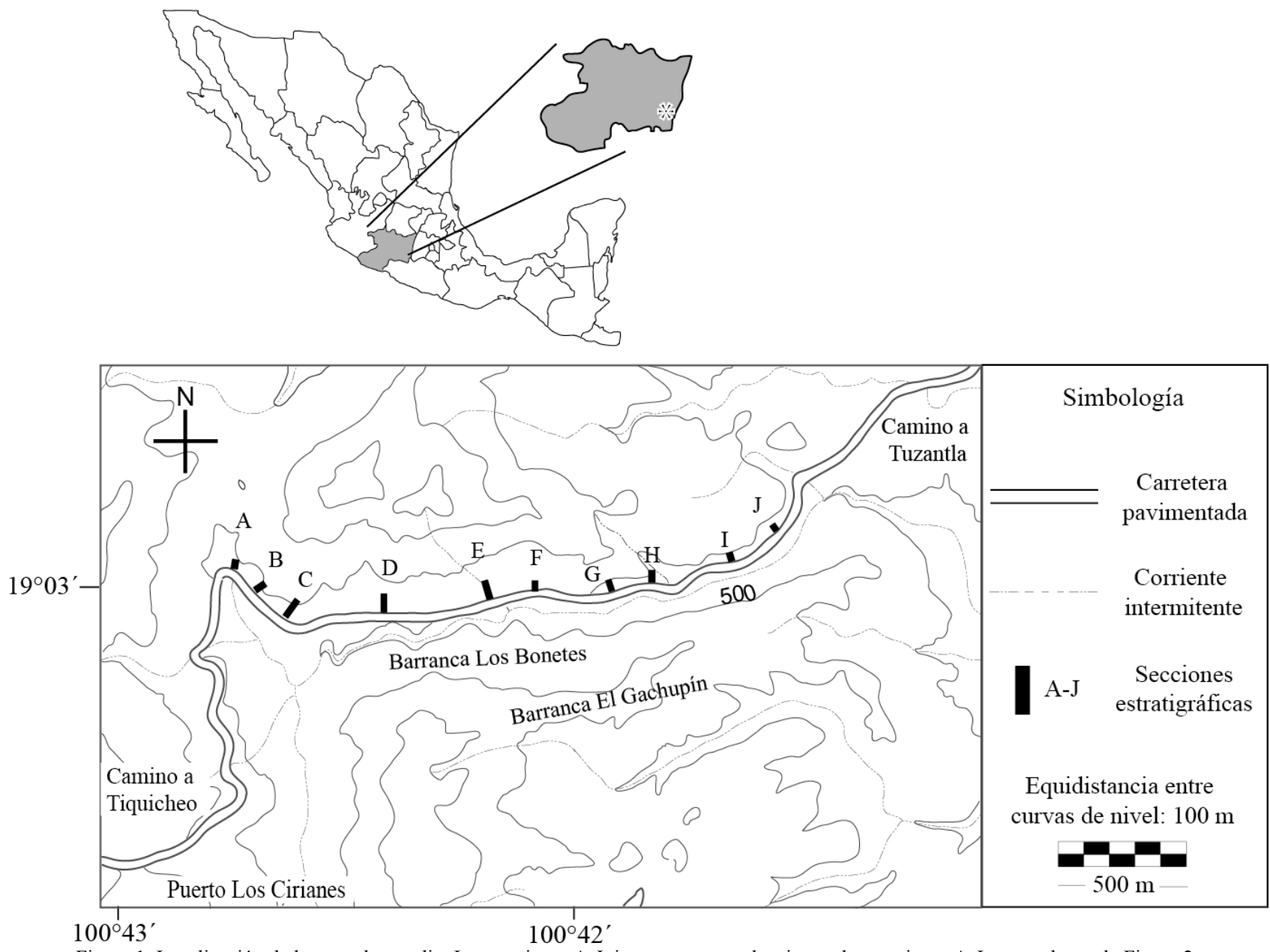

Figura 1. Localización de la zona de estudio. Las secciones A-J tienen correspondencia con las secciones A-J mostradas en la Figura 2. 
Centeno et al., 2003). El magmatismo presente tanto en éste como en la zona de estudio está íntimamente relacionado a los eventos de subducción de las placas del Pacífico Oriental que dieron origen a procesos de vulcanismo de composición calcialcalina, inicialmente submarino y posteriormente subaéreo. En el Cenozoico continuó el vulcanismo de arco que dio origen a la Sierra Madre Occidental (SMO).

Los arcos magmáticos precenozoicos relacionados con la subducción y la margen del Pacífico de México están mejor estudiados en los sectores norte (SonoraChihuahua) y centro (Sinaloa-Durango) de México. El sector sur (Nayarit-Jalisco-Zacatecas) ha sido menos estudiado debido a la escasez de afloramientos de dicho arco, lo cual puede deberse a la extensa cobertura de las ignimbritas del Oligoceno y del Mioceno temprano (Ferrari y Cerca-Martínez, 2005). Schaaf et al. (1995) mencionan la ocurrencia de un arco magmático cretácico en México sobre el Margen Activo Pacífico desde hace 100 Ma hasta hace $40 \mathrm{Ma}$.

Sin embargo, poco se conoce sobre la estratigrafía, sedimentología y ambientes de depósito del área de estudio; el único trabajo previo que existe es el de Altamira-Areyán (2002). Dicho autor propone 5 asociaciones de facies para el área de El Limón de Papatzingán, dentro de la cual se incluye la zona de estudio, y describe a Los Bonetes como constituida por conglomerados soportados por matriz y por clastos, brechas volcánicas y tobas, areniscas medias sin estructuras y horizontes de caliza y limolita, la cual fue interpretada como barras longitudinales de ríos, intercaladas con depósitos de brechas volcánicas (derrames y tobas líticas) y depósitos de lahar, que fueron cubiertos por depósitos de abanico de desborde sobre los cuales se desarrollaron suelos.

\section{Métodos}

Se realizaron 4 salidas a la zona de estudio para levantar la sección estratigráfica. El levantamiento de la sección fue continuo a lo largo del tramo de la carretera federal No. 51 que une a los municipios de Tuzantla y Tiquicheo, sin embargo hubo trayectos que no pudieron ser analizados debido a vegetación exuberante o derrumbes. El tamaño total de la sección es de alrededor de $392 \mathrm{~m}$. Los datos analizados en campo fueron litología, forma de los estratos, contenido biótico, espesor, contactos y orientación de los estratos (rumbo y echado). Asimismo fueron recolectadas muestras de mano para su posterior análisis en lámina delgada.

Las láminas delgadas fueron hechas para uso petrográfico, es decir con un espesor de $0.03 \mathrm{~mm}$. Se analizaron con microscopio petrográfico bajo objetivos de 10x hasta 40x, usando luz polarizada y nicoles cruzados para ver los colores de interferencia de los minerales. Se analizó en ellas el tipo calcita presente, los minerales flotantes y el contenido biótico.

\section{Resultados}

Con base en las características sedimentarias fueron reconocidas 6 litofacies (Figura 2): areniscas sin estructuras, areniscas con laminación tangencial, areniscas con laminación planar, conglomerado soportado por matriz, conglomerado soportado por clastos y calcretas pedogenéticas. Las areniscas son principalmente feldespáticas y litarenitas feldespáticas. Los feldespatos corresponden a feldespatos potásicos y plagioclasas. Los fragmentos líticos son de origen volcánico y sedimentario. El cuarzo, que se presenta en cantidades reducidas, es volcánico. Algunos paquetes de sedimentos finos contienen elementos esqueléticos desarticulados de dinosaurios, principalmente hadrosaurios, aunque su representatividad, hasta el momento, es baja.

Ambos conglomerados, el conglomerado soportado por matriz y el conglomerado soportado por clastos, tienen una pobre selección de clastos, son de composición volcánica, presentan ausencia de estructuras sedimentarias y están embebidos en una matriz fina.

En el caso de las calcretas, de acuerdo a las características macro- y microestructurales, fueron identificados dos tipos de acuerdo a su grado de evolución y lo descrito por Klappa (1983), Netterberg y Caiger (1983) y Alonso-Zarza (2003). Las calcretas se encuentran principalmente distribuidas desde la parte inferior hasta la parte media de la sección estratigráfica (Figura 2) y regularmente están intercaladas con paquetes de areniscas sin estructuras. La descripción de las calcretas se basa en la terminología de FitzPatrick (1984), Bullock et al. (1985) y Goudie (1996).

\subsection{Calcretas tipo I}

Las calcretas tipo I (Figura 3) corresponden a capas continuas de espesores variables entre 60 y $76 \mathrm{~cm}$. La forma de la capa varía entre planar y ondulosa. Se trata de suelos apedales con una gran cantidad de trazas verticales que aquí son interpretadas como raíces (Figura 3C). Dentro de la microestructura es muy prominente la textura alveolar donde los canales (Figura 3D) han sido sustituidos principalmente por calcita romboedral y fisuras curvadas y rectas. Las fisuras son escasas en las muestras y no en todas están presentes, contrario a lo que sucede con los canales que son muy abundantes en todas las muestras. Las fábricas son inequigranulares de tipo porfiriotópica, cuyos componentes minerales consisten principalmente en calcita microcristalina (micrita y microespatita) y calcita romboedral. Existen algunos componentes flotantes correspondientes a fragmentos líticos volcánicos, cuarzo y feldespatos en porcentajes menores al $10 \%$. Se observan algunas fisuras cubiertas, canales de Fe-Mn, así como elementos biogénicos. Los elementos biogénicos incluyen rizolitos, filamentos calcificados, restos de tejido celular y un corte de alguna raíz o tallo de planta vascular no identificable 
Calcretas pedogénicas en el Cretácico Tardio de Michoacán, México

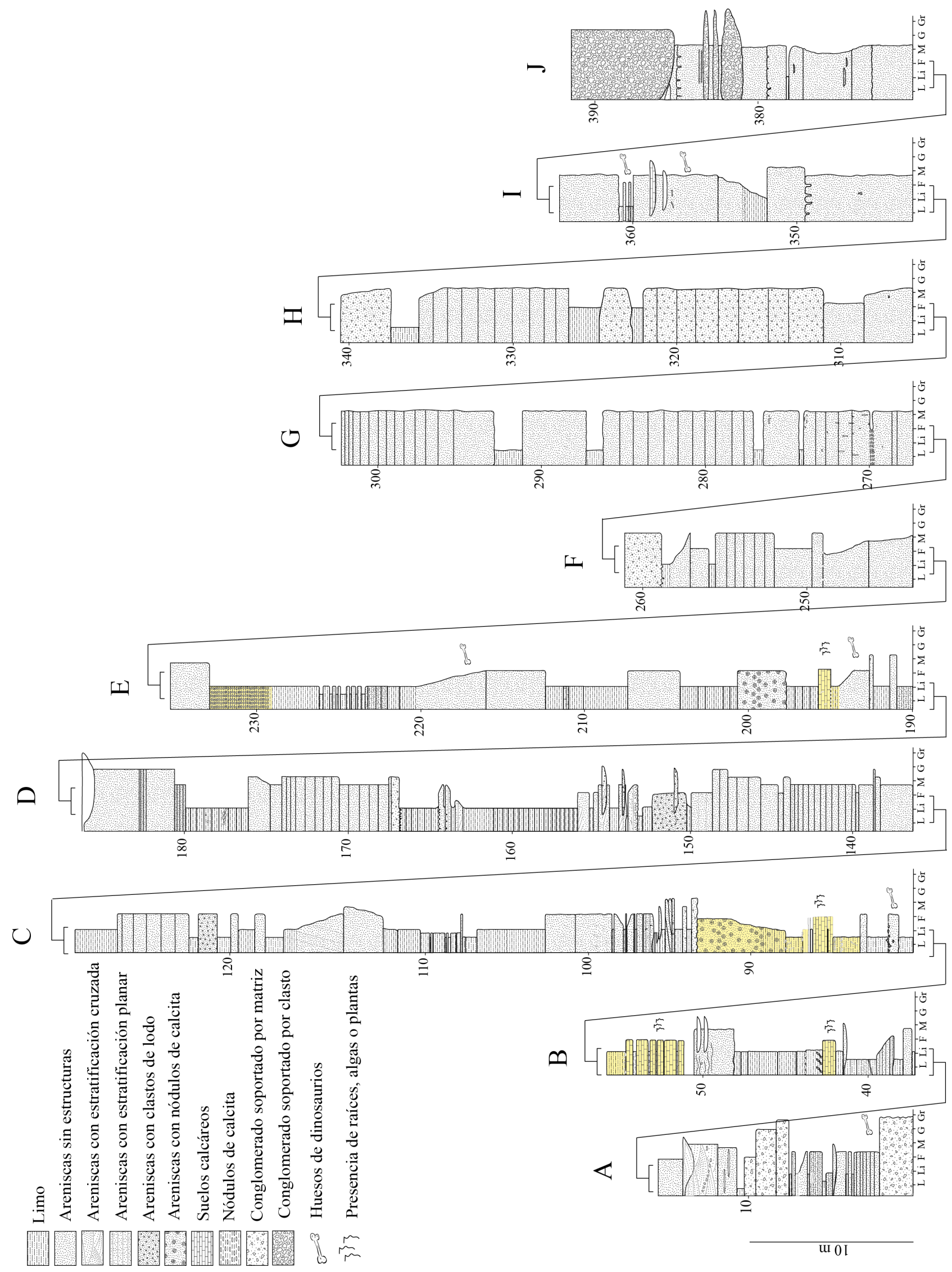

Figura 2. Sucesión sedimentaria levantada a lo largo de la carretera federal No. 51. Las letras se corresponden con las de la Figura 1. $\mathrm{L}=$ lodo; $\mathrm{Li}=$ limo; $\mathrm{F}=$ areniscas finas; $\mathrm{M}=$ areniscas medias; $\mathrm{G}=$ areniscas gruesas; $\mathrm{Gr}=$ gravas. En amarillo se resaltan las unidades pedoestratigráficas. 


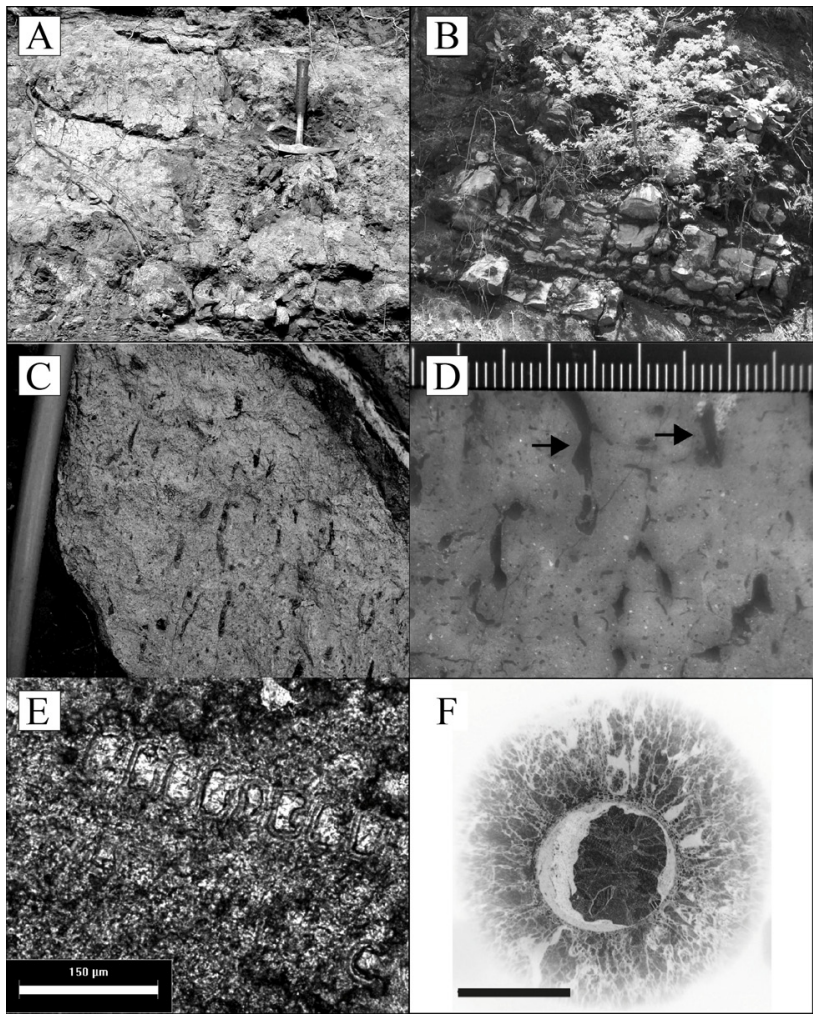

Figura 3. Calcretas y estructuras presentes en la Barranca Los Bonetes. A. Calcreta tabular. B. Calcretas intercaladas con lodos. C. Trazas de raíces presentes en las calcretas. D. Estructuras de canal. Cada línea representa $1 \mathrm{~cm}$. E. Presencia de una probable alga o cianobacteria debido al tipo de pared celular. F. Raíz o tallo de una planta vascular. La línea representa $1 \mathrm{~cm}$.

debido a la falta del estele (Figuras 3E y 3F). Hay presencia de revestimientos de arcillas y de calcita alrededor de granos individuales. Algunos granos han sido reemplazados con calcita romboedral.

En algunas ocasiones se observan lodos calcáreos intercalados entre las capas cuyas dimensiones varían entre 20 y $50 \mathrm{~cm}$. Los lodos están altamente cementados con calcita y presentan rompimiento concoidal. Dentro de la microestructura se observan canales que tienden a bifucarse y de los cuales la mayoría termina en bordes redondeados. La matriz está compuesta de micrita así como granos flotantes correspondientes a feldespatos y fragmentos líticos, agregados y granos envueltos. Hay presencia de calcita acicular y espatita rellenando cavidades. Las evidencias biogénicas incluyen filamentos calcificados y rizolitos.

\subsection{Calcretas tipo II}

Las calcretas tipo II se presentan como horizontes nodulares (Figura 4). El espesor varía entre 60 y $100 \mathrm{~cm}$. Presentan nódulos de calcita embebidos en una matriz lodosa. Los nódulos son oblicuos y corresponden a tallas de 3 a $10 \mathrm{~cm}$ sin ningún patrón de arreglo dentro del horizonte. En lámina delgada los constituyentes principales

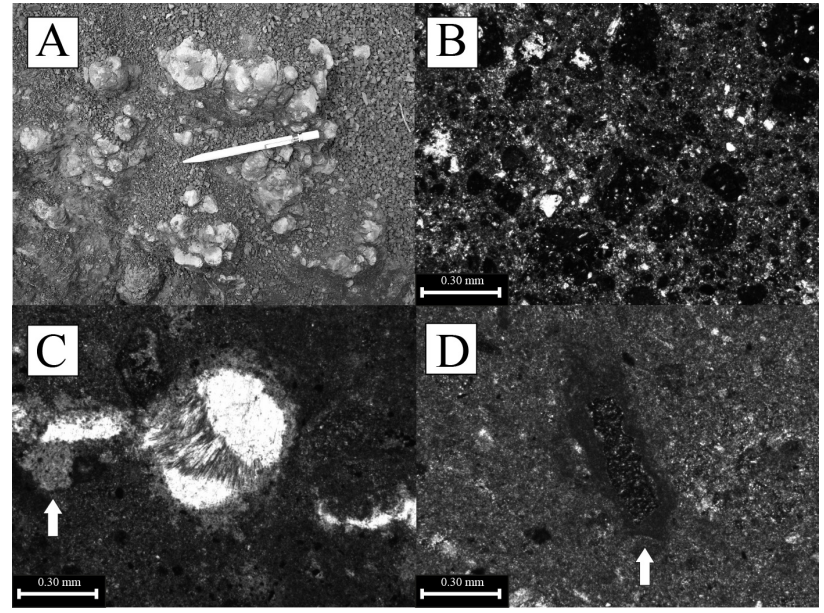

Figura 4. Calcretas nodulares. A: Acercamiento a los nódulos. B: Agregados. C: Revestimientos de arcilla alrededor de granos de calcita. Obsérvese que sobre un grano de calcita crece calcita acicular. D: Revestimiento de arcillas de un grano volcánico. Las flechas en C y D señalan las coberturas de arcilla.

son la micrita y la microespatita. Se observan agregados de material fino de milímetros de espesor con revestimientos de calcita (Figuras 4C y 4D). Existen granos flotantes de feldespatos, fragmentos líticos y cuarzo en bajas proporciones; muchos de ellos han sido reemplazados por calcita romboedral. Hay presencia de fábrica alveolar y de calcita acicular. No hay presencia de elementos orgánicos.

En todas las láminas delgadas es común observar espatita creciendo sobre los fragmentos minerales y reemplazo de las estructuras orgánicas por calcita.

\section{Discusión}

5.1. Mecanismos de formación de las calcretas de la barranca Los Bonetes

Las facies predominantes en el área de estudio corresponden a areniscas sin estructuras y conglomerados soportados por matriz, interpretadas como flujos sedimentarios gravitacionales arenosos y detríticos, respectivamente. Debido a que la composición de los clastos es volcánica, los flujos sedimentarios gravitacionales son interpretados como lahares, siendo los procesos predominantes en laderas volcánicas y que recorren grandes distancias. Por lo anterior, se asume que la sucesión sedimentaria de la barranca Los Bonetes fue en la zona distal de un volcán.

El depósito no fue constante. Hubo periodos de altas tasas de sedimentación, suposición hecha con base en la abundancia de flujos sedimentarios gravitacionales, seguida por periodos de no depósito debido a la presencia de bioturbadores y paleosuelos (calcretas). Las calcretas pueden tardar en formarse desde miles hasta millones de 
años (Scharpensseel y Targulian, 1990; Alonso-Zarza, 2003).

Las calcretas presentes en la barranca Los Bonetes son consideradas de origen pedogenético debido a la presencia de texturas alfa y beta, al arreglo de sus capas y sus estructurales macroscópicas como son las trazas de raíces. El proceso pedogenético predominante fue la acumulación de calcita y, sólo en algunos casos y en proporciones menores al $5 \%$, procesos de óxido-reducción, que en lámina delgada se observa que son una etapa posterior a la acumulación de calcita debido a que crecen sobre ésta. Con base en todas las evidencias anteriormente descritas, el tipo de horizonte presente es Bk en distintas etapas de evolución.

Las características macro- y micromorfológicas de las calcretas permiten asignarlas a los siguientes tipos: nodular, trenzado y de parrilla. Estos distintos tipos de calcretas responden a los distintos grados de evolución de las mismas (Klappa, 1983; Alonso-Zarza, 2003; Figura 5).

La calcreta tipo I tiene una variación entre calcretas trenzadas y horizontes parrilla. Las calcretas trenzadas se reconocen debido a la forma ondulosa de sus capas y microscópicamente por la abundancia de texturas alveolares y rizolitos. A la base de los mismos se observa una textura colmenar de 2 a $3 \mathrm{~cm}$ de espesor, como la descrita por Netterberg y Caiger (1983) para este tipo de horizontes. Sin embargo, difiere de las observaciones hechas por Klappa (1983), Netterberg y Caiger (1983) y Alonso-Zarza (2003), en que solamente en dos casos suprayace a las calcretas nodulares; en los otros dos casos suprayace a un horizonte de lodos calcáreos. Sin embargo, como menciona Alonso-Zarza (2003), los perfiles de las calcretas pueden ser relativamente complejos, con horizontes similares ocurriendo en distintas posiciones dentro de la calcreta, lo que indica perfiles

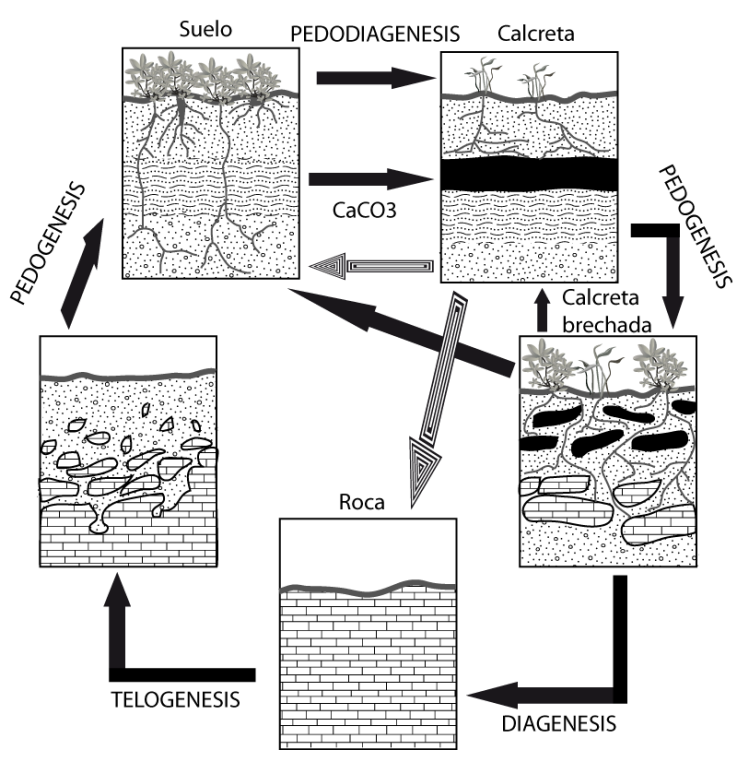

Figura 5. A. Ciclo pedo-diagenético. B. Suelo idealizado de la formación de un perfil calcáreo. Modificado de Klappa (1983). compuestos. En contraste, horizontes significativos pueden estar ausentes debido a truncamientos dentro del desarrollo del perfil.

Los horizontes parrilla, de acuerdo a Klappa (1983), Netterberg y Caiger (1983) y Alonso-Zarza (2003), representa suelos maduros. En la zona de estudio sólo fue observado un horizonte parrilla ya que está altamente endurecido y se encuentra compuesto por micrita. Suprayace el horizonte trenzado.

La calcreta tipo II es reconocida como un horizonte nodular debido a la presencia de nódulos de carbonato de calcio embebidos en una matriz lodosa y a que la matriz de los nódulos es micrítica.

En la barranca Los Bonetes se observan dos secuencias distintas de calcretas pedogenéticas con base en su grado de madurez y arreglo. La primera secuencia (Figura 6) presenta todas las unidades mencionadas en el perfil de Klappa (1983), lo cual indica periodos de emergencia que permitieron el desarrollo del suelo hasta sus etapas maduras, como se evidencia por la presencia del horizonte parrilla (Klappa, 1983; Alonso-Zarza, 2003). Este horizonte presenta una gran cantidad de evidencia biogénica, principalmente raíces.

La segunda secuencia de calcretas presenta una serie de intercalaciones entre lodos calcáreos y calcretas trenzadas (Figura 7), lo cual indicaría que tuvo varias etapas de formación o que los procesos de intemperismo pudieron eliminar algunos horizontes dentro de la sección.

La fuente de calcio para el desarrollo de las calcretas fueron los minerales primarios contenidos en los sedimentos volcánicos, que presentan proporciones variables de $\mathrm{CaO}$
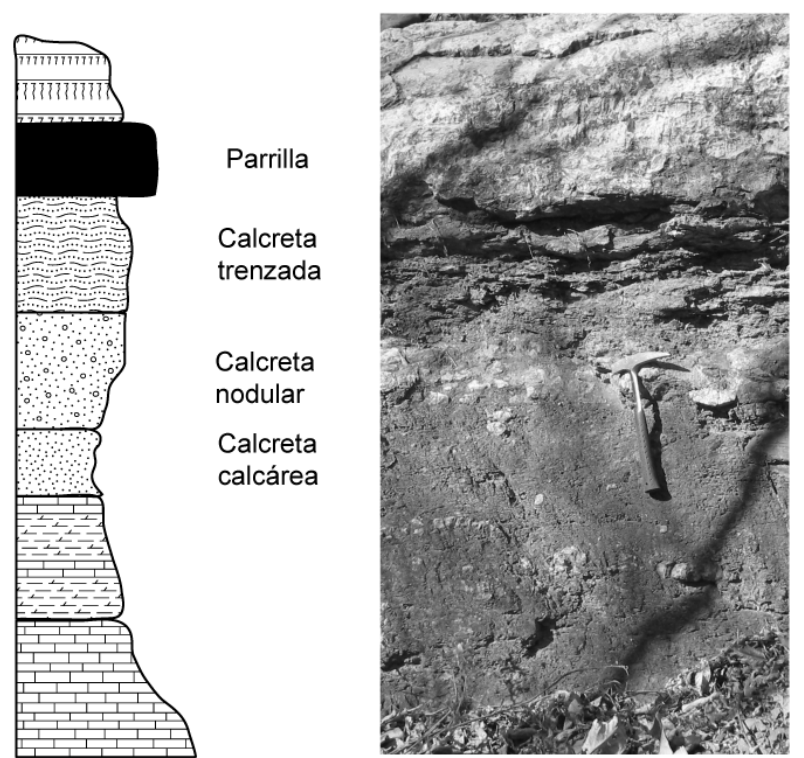

Figura 6. Horizonte ideal de Klappa (1983) a la izquierda. A la derecha, horizonte presente en la Barranca Los Bonetes. Se puede observar que existe una correspondencia entre las calcretas del modelo de Klappa (1983) y las presentes en la zona de estudio, indicándose el tipo de calcreta que presenta. 


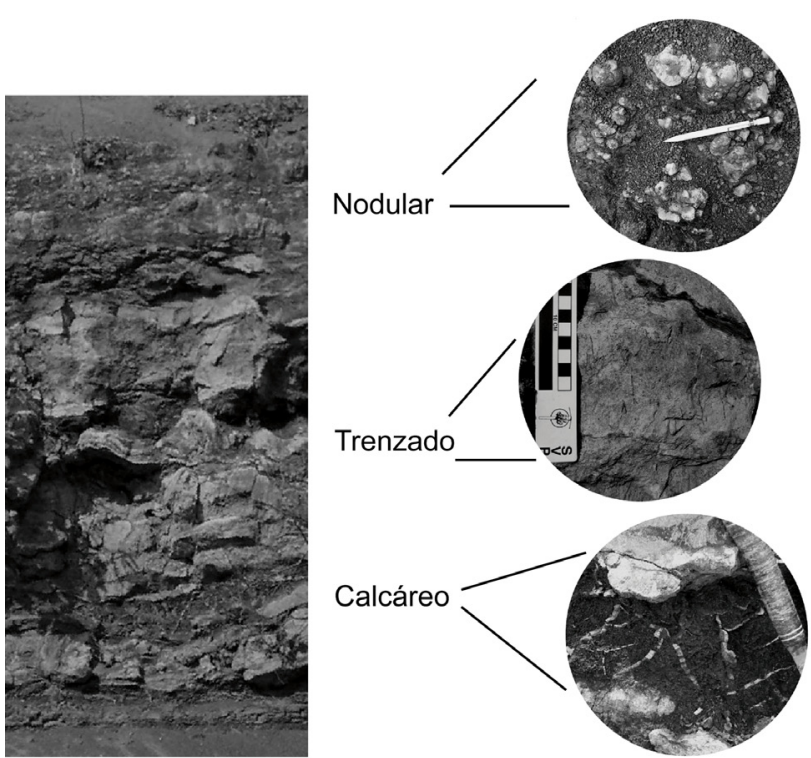

Figura 7. Secuencia de calcretas con intercalaciones entre lodos calcáreos y horizontes trenzados; hasta la cima un horizonte nodular. A la derecha se observan algunos acercamientos de los distintos paquetes.

(McBirney, 1993). El calcio se libera principalmente por la alteración de las plagioclasas, del vidrio volcánico y de los fragmentos líticos (Surdam y Boles, 1979). Otras fuentes externas que pudieron proveer de calcio al medio son el agua superficial y el agua de lluvia, plantas que contienen calcio en sus tejidos y la desintegración de conchas de moluscos. De hecho, la presencia de filamentos calcificados (Figura 3E) demuestra su contribución en la formación de carbonato de calcio (Klappa, 1983).

Las calcretas presentes en la barranca Los Bonetes se debieron formar con base en los siguientes procesos: 1) precipitación y acumulación de carbonatos a través del relleno de poros, principalmente de los conductos de las raíces y fisuras presentes; 2) precipitación de nódulos en el suelo; 3) precipitación y acumulación continua dando lugar a un horizonte laminar; 4) foliación y brechamiento de los horizontes.

\subsection{Paleoambiente de la secuencia de la Barranca de los Bonetes}

Anteriormente la sucesión volcaniclástica de la barranca Los Bonetes había sido interpretada como depósitos de abanico de desborde (Altamira-Areyán, 2002), sin embargo, no existen evidencias que apoyen esta idea. Los depósitos de abanico de desborde son minideltas que se acrecionan dentro de la planicie de inundación debido al rompimiento del banco del canal (Miall, 1992). Se caracterizan por la presencia de areniscas laminadas y areniscas con estratificación cruzada debido a la migración de dunas (O’Brien y Wells, 1986). Están compuestos por secuencias granocrecientes de areniscas y lodos que son de 2 a $3 \mathrm{~m}$ de grosor y que se extienden por cientos de metros. Presentan elementos deposicionales asociados, tales como canales, diques y barras de boca (Bridge, 2003). Ninguna de estas características está presente en la zona de estudio, contrario a lo que sucede con los procesos y depósitos asociados a los volcanes en sus partes más distales.

Asimismo, Altamira-Areyán (2002) determinó las calcretas como de origen lacustre, lo cual es poco factible debido a que los cementos carbonatados pedogenéticos se forman principalmente como una fase de relleno de poros mientras que los lacustres se forman por precipitación (Chafetz et al., 1985). De hecho, no observamos ninguna evidencia de sedimentación lacustre como estructuras sedimentarias o facies (por ejemplo, Talbot y Allen, 2007).

El comportamiento observado en las muestras en lámina delgada es sustitución de granos, por lo cual se asocia a un origen pedogenético. El segundo argumento es la presencia de raíces, filamentos calcificados y probables heces. Los filamentos calcificados pueden pertenecer a bacterias, algas, hongos, líquenes o posiblemente a los filamentos de las raíces secundarias de las plantas vasculares; sin embargo, su identificación es difícil (Goudie, 1996).

Para poder determinar el clima, Altamira-Areyán (2002) obtuvo muestras de polen de los horizontes trenzados, que fueron analizadas por el Dr. Enrique Martínez y la M. en C. Elia Ramírez, mencionando que la asociación palinológica (Pseudoplicapollis sp., Myrtaceoipollenites sp., Nudopollis sp., Trudopollis pertrudens, Momipites microcoriphaeus, Caryapollenites sp. y Momipites aff. tenuipolus) corresponde a asociaciones autóctonas con poco o nulo transporte, sugiriendo una temperatura calurosa de clima paratropical, donde prevaleció el bosque deciduo a elevaciones aproximadas de mil metros sobre el nivel del mar (Altamira-Areyán et al., 2000). Sin embargo, los resultados obtenidos en este trabajo indican que los suelos son de tipo cálcico (calcretas), los cuales han sido ampliamente reportados en suelos áridos o semiáridos (Netterberg y Caiger, 1983; Collinson, 1996; Goudie, 1996; Birkeland, 1999; Alonso-Zarza, 2003).

Los suelos son formados por intemperismo y transformación del material parental in situ o por el intemperismo y/o transformación del material transportado derivado de rocas sólidas. Los procesos formadores del suelo (clima, biota, topografía, material parental y tiempo) causan modificaciones en el sedimento o roca intemperizada por remoción, translocación, transformación y neoformación de constituyentes móviles (Simonson, 1959). Estos cambios generalmente producen diferenciación en horizontes y la formación del perfil del suelo (Klappa, 1983), reflejando las características ambientales del lugar.

Las calcretas se localizan comúnmente en los abanicos aluviales en conjuntos áridos o semiáridos debido a que los bajos volúmenes de agua que penetran el suelo no tienden a infiltrase sino que se pierden a través de evaporación o transpiración. El crecimiento de las plantas es poco abundante y la oxidación más rápida, por tanto la acumulación de la materia orgánica es reducida (Collinson, 
1996), como se ha observado en las calcretas presentes en la sucesión sedimentaria de la barranca Los Bonetes.

Por todo lo anteriormente expuesto, se infiere que el clima local predominante en la zona de estudio debió ser semiárido. El clima paratropical determinado a través de palinomorfos (Altamira-Areyán et al., 2000) para la zona de estudio no es muy confiable debido a que el polen puede recorrer grandes distancias, aunando al hecho de la baja representatividad del polen en el área de estudio y a que no hay otro tipo de evidencias que apoyen este tipo de clima en la región, como sería la presencia de maderas.

\section{Conclusiones}

Los paleosuelos han mostrado ser una herramienta confiable en la determinación climática local o a escala pequeña en una región a través de horizontes marcadores, siendo aún más confiables que otras evidencias como son los palinomorfos y los fósiles de vertebrados o plantas, ya que los paleosuelos se forman in situ y a través de sus procesos de formación se puede conocer el ambiente en el que se formaron.

El análisis de las calcretas presentes en la sucesión sedimentaria de la barranca Los Bonetes indicó que durante los periodos de no depósito se desarrollaron suelos con alta acumulación de calcita que son característicos de las regiones áridas y semiáridas del mundo.

La importancia de este trabajo radica en que es el único estudio que existe en el país que analiza paleosuelos precuaternarios.

\section{Agradecimientos}

Los autores agradecen el financiamiento otorgado por National Geographic Society (Grant no. n7953-05) para la realización de este trabajo, al Instituto de Geología y a la Universidad de Rhode Island por permitirnos hacer uso de sus instalaciones y equipo. Al poblado de Tiquicheo por toda la ayuda prestada durante las diferentes temporadas de campo.

\section{Referencias}

Alonso-Zarza, A.M., 2003, Palaeoenvironmental significance of palustrine carbonates and calcretes in the geological record: Earth-Science Reviews, 60, 261-298.

Altamira-Areyán, A., 2002, Las litofacies y sus implicaciones de la cuenca sedimentaria Cutzamala-Tiquicheo, Estados de Guerrero y Michoacán, México: Ciudad de México, Instituto de Geología, Universidad Nacional Autónoma de México, tesis de maestría, 79 p.

Altamira-Areyán, A., Martínez-Hernández, E., Centeno-García, E., 2000, Facies, paleoambientes y paleoclimas del Paleoceno-Eoceno de la zona norte de Tierra Caliente, estados de Michoacán y Guerrero (resumen), en Segunda Reunión Nacional de Ciencias de la Tierra:
Puerto Vallarta, Jalisco, México, Unión Geofísica Mexicana, 157. Benammi, M., Centeno-García, E., Martínez-Hernández, E., MoralesGámez, M., Tolson, G., Urrutia-Fucugauchi, J., 2005, Presencia de dinosaurios en la barranca Los Bonetes en el sur de México (región de Tiquicheo, estado de Michoacán) y sus implicaciones cronoestratigráficas: Revista Mexicana de Ciencias Geológicas, $22,429-435$.

Birkeland, P.W., 1999, Soils and Geomorphology: New York, Oxford University Press, $430 \mathrm{p}$.

Bridge, J.S., 2003, Rivers and Floodplains. Forms, Processes, and Sedimentary Record: Oxford, R.U., Blackwell Publishing, 491 p.

Buick, R., Thornett, J.R., McNaughton, N.J., Smith, J.B., Barley, M.E., Savage, M., 1995, Record of emergent continental crust approximately 3.5 billion years ago in the Pilbara craton of Australia: Nature, 375, 574-577.

Bullock, P., Fedoroff, N., Jongerus, A., Stoops, G., Tursina, T., Babel, U., Aguilar, J. Altemüller, H.J., FitzPatrick, E.A., Kowalinski, St., Paneque, G., Rutherford, G.K., Yarilova, E.A., 1985, Handbook for soil thin section description: Albrighton, Wolverhampton, R.U., Waine Research Publications, $152 \mathrm{p}$.

Centeno-García, E., Corona-Chávez, P., Talavera-Mendoza, O., Iriondo, A., 2003, Geology and tectonic evolution of the western Guerrero terrane - a transect from Puerto Vallarta to Zihuatanejo, México, en Geologic Transects Across Cordilleran Mexico: Guidebook for the field trips of the $99^{\text {th }}$ Geological Society of America Cordilleran Section Annual Meeting: Ciudad de México, Instituto de Geología, Universidad Nacional Autónoma de México, 201-228.

Chafetz, H.S., Wilkinson, B.H., Love, K.M., 1985, Morphology and composition of non-marine carbonate cements in near-surface settings, en Schneidermann, N., Harris, P.M. (eds.), Carbonate Cements: Tulsa, Oklahoma, Society of Economic Paleontologists and Mineralogists, 337-347.

Collinson, J.D., 1996, Alluvial sediments, en Reading, H.G. (ed.), Sedimentary Environments: Processes, Facies and Stratigraphy: Oxford, R.U., Blackwell Publishing, 37-82.

Ferrari, L., Cerca-Martínez, M., 2005, El magmatismo Cretácico Tardío - Terciario temprano del Sur y occidente de México: implicaciones tectónicas: Geos, 25(1), 98.

FitzPatrick, E.A., 1984, Micromorphology of Soils: Londres, Chapman and Hall, $433 \mathrm{p}$.

Ghosh, P., Ghosh, P., Bhattacharya, S.K., 2001, $\mathrm{CO}_{2}$ levels in the Late Palaeozoic and Mesozoic atmosphere from soil carbonate and organic matter, Satpura Basin, Central India: Palaeogeography, Palaeoclimatology, Palaeoecology, 170, 219-236.

Goudie, A.S., 1996, Review: Organic agency in calcrete development: Journal of Arid Environments, 32, 103-110.

Jenny, H., 1941, Factors of Soil Formation: A System of Quantitative Pedology: New York, McGraw-Hill, 281 p.

Klappa, C.F.A., 1983, A process-response model for the formation of pedogenic calcretes, en Wilson, R.C.L. (ed.), Residual Deposits: Surface Related Weathering Processes and Materials: Londres, Blackwell Scientific Publications, 211-220.

Košir, A., 2004, Microcodium revisited: root calcification products of terrestrial plants on carbonate-rich substrates: Journal of Sedimentary Research, 74, 845-857.

Kraus, M.J., 1999, Paleosols in clastic sedimentary rocks: their geologic applications: Earth-Science Reviews, 47, 41-70.

Krull, E.S., Retallack, G.J., 2000, $\delta^{13} \mathrm{C}$ depth profiles from paleosols across the Permian-Triassic boundary: Evidence for methane release: GSA Bulletin, 112, 1459-1472.

Lee, Y.W., Lee, Y.I., Hisada, K., 2003, Paleosols in the Cretaceous Goshoura and Mifune groups, SW Japan and their paleoclimate implications: Palaeogeography, Palaeoclimatology, Palaeoecology, 199, 265-282.

Mack, G.H., James, W.C., Monger, H.C., 1993, Classification of paleosols: Geological Society of America Bulletin, 105, 129-136.

McBirney, A.R., 1993, Igneous Petrology: Boston, Jones and Bartlett Publishers, 508p. 
Meyer, R., 1997, Paleoalterites and Paleosols. Imprints of Terrestrial Processes in Sedimentary Rocks: Rotterdam, A. A. Balkema Publishers, $162 \mathrm{p}$.

Miall, A.D., 1992, Alluvial deposits, en Walker, R.G., James, N.P. (eds.), Facies Models: Toronto, Canadá, Geological Association of Canada, 119-142.

Netterberg, F., Caiger, J.H.A., 1983, A geotechnical classification of calcretes and other pedocretes, en Wilson, R.C.L. (ed.), Residual Deposits: Surface Related Weathering Processes and Materials: Londres, Blackwell Scientific Publications, 235-243.

Nordt, L., Atchley, S., Dworkin, S.I., 2002, Paleosol barometer indicates extreme fluctuations in atmospheric $\mathrm{CO}_{2}$ across the CretaceousTertiary boundary: Geology, 30, 703-706.

O'Brien, B.J., Stout, J.D., 1978, Movement and turnover of soil organic matter as indicated by carbon isotope measurements: Soil Biology Biochemistry, 10, 309-317.

O’Brien, P.E., Wells, A.T., 1986, A small, alluvial crevasse splay: Journal of Sedimentary Petrology, 56, 876-879.

Ortiz-Hernández, L.E., Flores-Castro, K., Acevedo-Sandoval, O.A., 2002, Petrographic and geochemical characteristics of upper Aptian calcalkaline volcanism in San Miguel de Allende, Guanajuato state, Mexico: Revista Mexicana de Ciencias Geológicas, 19, 81-90.

Paik, I.S., Kim, H.J., Park, K.H., Song, Y.S., Lee, Y.I., Hwang, J.Y., Huh, M., 2001, Palaeoenvironments and taphonomic preservation of dinosaur bone-bearing deposits in the Lower Cretaceous Hasandong Formation, Korea: Cretaceous Research, 22, 627-642.

Rasbury, E.T., Gierlowsky-Kordesh, E.H., Cole, J.M., Sookdeo, C., Spataro, G., Nienstedt, J., 2006, Calcite cement stratigraphy of a nonpedogenic calcrete in the Triassic New Haven Arkose (Newark Supergroup), en Alonso-Zarza, A.M., Tanner, L.H. (eds), Paleoenvironmental record and applications of calcretes and palustrine carbonates: Boulder, Colorado, Geological Society of America, Special Paper 416, 203- 221.

Retallack, G.J., 1985, Fossil soils as grounds for interpreting the advent of large plants and animals on land: Philosophical Transactions of the Royal Society of London B, 309, 105-142.

Retallack, G.J., 2001, Soils of the Past: An Introduction to Paleopedology: Oxford, R.U., Blackwell Science, 404 p.

Retallack, G.J., James, W.C., Mack, G.H., Monger, H.C., 1993, Classification of paleosols: Discussion and reply: Geological Society of America Bulletin, 105, 1635-1637.

Retallack, G.J., Sheldon, N.D., Cogoini, M., Elmore, R.D., 2003, Magnetic susceptibility of early Paleozoic and Precambrian paleosols: Palaeogeography, Palaeoclimatology, Palaeoecology, 198, 373-380.
Schaaf, P., Morán-Zenteno, D., Hernandez-Bernal, M.S., Solis-Pichardo, G., Tolson, G., Köhler, H., 1995, Paleogene continental margin truncation in southwestern Mexico: Geochronological evidence: Tectonics, 14, 1339-1350.

Scharpensseel, H.W., Targulian, V.O., 1990, Types of soil processes and changes, en Arnold, R.W., Szabolcs, I., Targulian, V.O. (eds.), Global soil change: Laxenburg, Austria, International Institute for Applied Systems Analysis, 41-62.

Simonson, R.W., 1959, Outline of a generalized theory of soil genesis: Soil Science Society of America Journal, 23, 152-156.

Sreenivas, B., Roy, A.B., Srinivasan, R., 2001, Geochemistry of sericite deposits at the base of the paleoproterozoic aravalli supergroup, Rajasthan, India: Evidence for metamorphosed and metasomatised precambrian paleosol: Journal of Earth System Science, 110, 39-61.

Stanley, S.M., 2008, Earth System History: Nueva York, W.H. Freeman, $551 \mathrm{p}$.

Surdam, R.C., Boles, J.R., 1979, Diagenesis of volcanic sandstones, en Scholle, P.A., Schluger, P.R. (eds.), Aspects of Diagenesis: Tulsa, Oklahoma, Society of Economic Paleontologists and Mineralogists, 227-242.

Talbot, M.R., Allen, P.A., 2007, Lakes, en Reading, H.G. (ed.), Sedimentary Environments: Processes, Facies and Stratigraphy: Oxford, R.U., Blackwell Publishing, 83-124.

Tovar, R.E., Fastovsky, D.E., Benammi, M., Centeno-García, E., 2009, A new locality of dinosaur bones in volcaniclastic sediments in Michoacán, México (resumen), en Geological Society of America, 2009 GSA Annual Meeting Abstracts and Program: Boulder, Colorado, 209.

Watts, N.L., 1980, Quaternary pedogenic calcretes from the Kalahari (southern Africa): mineralogy, genesis and diagenesis: Sedimentology, 27, 661-686.

Wright, V.P., Tucker, M.E., 1991, Calcretes: Oxford, Blackwell Scientific Publications, $352 \mathrm{p}$

Manuscrito recibido: Enero 10, 2011

Manuscrito corregido recibido: Octubre 30, 2011

Manuscrito aceptado: Diciembre 12, 2011 